\title{
Dopaminergic presynaptic modulation of nigral afferents: its role in the generation of recurrent bursting in substantia nigra pars reticulata neurons
}

\author{
José de Jesús Aceves, Pavel E. Rueda-Orozco, Ricardo Hernández, Víctor Plata, Osvaldo lbañez-Sandoval, \\ Elvira Galarraga and José Bargas*
}

División de Neurociencias, Instituto de Fisiología Celular, Universidad Nacional Autónoma de México, Distrito Federal México, México

\section{Edited by:}

James M. Tepper, Rutgers, The State University of New Jersey, USA

Reviewed by:

Christian Lee, New York University School of Medicine, USA

Carlos A. Paladini, University of Texas at San Antonio, USA

\section{${ }^{*}$ Correspondence:}

José Bargas, Division of Neuroscience, Institute of Cell Physiology, Universidad Nacional Autónoma de México, PO Box: 70-253, Distrito Federal México 04510, México.

e-mail: jbargas@ifc.unam.mx
Previous work has shown the functions associated with activation of dopamine presynaptic receptors in some substantia nigra pars reticulata (SNr) afferents: (i) striatonigral terminals (direct pathway) posses presynaptic dopamine $D_{1}$-class receptors whose action is to enhance inhibitory postsynaptic currents (IPSCs) and GABA transmission. (ii) Subthalamonigral terminals posses $D_{1}$ - and $D_{2}$-class receptors where $D_{1}$-class receptor activation enhances and $D_{2}$-class receptor activation decreases excitatory postsynaptic currents. Here we report that pallidonigral afferents posses $D_{2}$-class receptors ( $D_{3}$ and $D_{4}$ types) that decrease inhibitory synaptic transmission via presynaptic modulation. No action of $D_{1}$-class agonists was found on pallidonigral synapses. In contrast, administration of $\mathrm{D}_{1}$-receptor antagonists greatly decreased striatonigral IPSCs in the same preparation, suggesting that tonic dopamine levels help in maintaining the function of the striatonigral (direct) pathway. When both $D_{3}$ and $D_{4}$ type receptors were blocked, pallidonigral IPSCs increased in amplitude while striatonigral connections had no significant change, suggesting that tonic dopamine levels are repressing a powerful inhibition conveyed by pallidonigral synapses (a branch of the indirect pathway). We then blocked both $D_{1}$ - and $D_{2}$ class receptors to acutely decrease direct pathway (striatonigral) and enhance indirect pathways (subthalamonigral and pallidonigral) synaptic force. The result was that most SNr projection neurons entered a recurrent bursting firing mode similar to that observed during Parkinsonism in both patients and animal models. These results raise the question as to whether the lack of dopamine in basal ganglia output nuclei is enough to generate some pathological signs of Parkinsonism.

Keywords: basal ganglia, striatum, substantia nigra pars reticulata, dopamine function, striatonigral synapses, pallidonigral synapses, $D_{1}$-receptors, $D_{2}$-receptors

\section{INTRODUCTION}

The internal globus pallidus (GPi) and substantia nigra pars reticulata $(\mathrm{SNr})$ are the basal ganglia (BG) output nuclei. Besides projecting to the thalamus to form the cortico-BG loops (Chevalier et al., 1985; Albin et al., 1989; Smith and Bolam, 1989; Alexander and Crutcher, 1990; DeLong, 1990; Smith et al., 1998; Haber, 2003), output nuclei also project to pons and brain stem to control descending pathways and central patterns generators (CPGs) that regulate muscular tone and automatic or rhythmic motor responses (Takakusaki et al., 2003, 2004; Grillner et al., 2008). In birds, reptiles, and lower vertebrates in which the cortex is not well developed, the control of brain stem nuclei is a main function of the BG (Reiner et al., 1998; Grillner et al., 2005, 2008; Gale and Perkel, 2010). In the SNr, inhibitory postsynaptic currents (IPSCs) are in part provided by striatonigral direct pathway terminals (Grofová and Rinvik, 1970; Chevalier et al., 1985; Smith and Bolam, 1991; Deniau et al., 1996; Matuszewich and Yamamoto, 1999), which possess functional presynaptic dopamine $\mathrm{D}_{1}$-receptors whose activation increases direct pathway inhibition (Porceddu et al., 1986; Altar and Hauser, 1987; Floran et al., 1990; Radnikow and Misgeld, 1998; Chuhma et al., 2011). Enhancement of direct pathway inhibition facilitates movements while its reduction represses them (Albin et al., 1989; Bateup et al., 2010; Kravitz et al., 2010; Redgrave et al., 2010). In contrast, subthalamonigral afferents compose the last step of the indirect pathway (Nakanishi et al., 1987; Bevan et al., 1994). Presynaptic modulation of subthalamonigral terminals by dopamine uses both classes of dopamine receptors: $\mathrm{D}_{1}$ and $\mathrm{D}_{2}$ (Ibáñez-Sandoval et al., 2006). Activation of $\mathrm{D}_{1}$ enhances while activation of $\mathrm{D}_{2}$ depresses subthalamonigral excitatory postsynaptic currents (EPSCs). Interestingly, simultaneous blockade of both receptors induced larger evoked EPSCs, suggesting that $\mathrm{D}_{2}$-receptors have more influence than $\mathrm{D}_{1}$-receptors in the modulation of transmission (Ibáñez-Sandoval et al., 2006).

In parallel, the external globus pallidus ( $\mathrm{GPe}$ ) also sends an inhibitory input to SNr (Bevan et al., 1996; Kita, 2007; Connelly et al., 2010). In the present work we investigated dopaminergic presynaptic modulation of inhibitory pallidonigral afferents. We found that $\mathrm{D}_{2}$-class receptors regulate these terminals with no sign of $\mathrm{D}_{1}$-receptor modulation as compared to striatonigral $\mathrm{D}_{1}$-mediated modulation in the same preparation. Interestingly, and as shown before for subthalamonigral afferents (Ibáñez-Sandoval et al., 2006), we found that addition of selective receptor antagonists disclose a tonic action of ambient dopamine levels on both, striatonigral and pallidonigral afferents, supporting the notion that dopamine 
receptors are sensing extracellular dopamine continuously (Yanovsky et al., 2003). Furthermore, the blockade of all dopamine receptor types altogether induced a recurrent bursting firing pattern in SNr projection neurons, a mode of firing typically seen in both Parkinsonian humans and animals (e.g., Magill et al., 2001; Walters et al., 2007; Walters and Bergstrom, 2009; Zold et al., 2009). This finding indicates that it is enough to acutely block dopamine receptors to induce a Parkinsonian firing pattern in SNr neurons. Given the direct projection of SNr to brain stem nuclei controlling muscular tone and CPGs activation, a question as to whether dopamine dysfunction in the output nuclei is enough to produce some Parkinsonian signs (Morris et al., 1994; Hemsley and Crocker, 1998; Hikosaka et al., 2000; Takakusaki et al., 2003) is set forth.

\section{MATERIALS AND METHODS}

Procedures were carried out in accordance with the National Institutes of Health Guide for Care and Use of Laboratory Animals (NIH Publications No. 8023, revised 1996) and were approved by the Institutional Animal Care Committee of UNAM. Methods have been reported elsewhere (Beurrier et al., 2006; Ibáñez-Sandoval et al., 2006). Briefly, Wistar rats (15-40 postnatal day), were anesthetized with isoflurane, decapitated, and their brains removed. Parasagittal or parahorizontal slices $(300 \mu \mathrm{m})$ containing the neostriatum (NSt), globus pallidus (GP), and substantia nigra pars reticulata $(\mathrm{SNr})$ were obtained with a vibratome in saline of the following composition (in millimolar): 124 choline chloride, 2.5 $\mathrm{KCl}, 1.0 \mathrm{MgCl}_{2}, 1.2 \mathrm{NaH}_{2} \mathrm{PO}_{4}, 2.0 \mathrm{CaCl}_{2}$, and 10 glucose $\left(\sim 4^{\circ} \mathrm{C} 95 \%\right.$ $\left.\mathrm{O}_{2}, 5 \% \mathrm{CO}_{2}\right)$. Whole-cell patch-clamp recordings were performed on rat SNr neurons (Ibáñez-Sandoval et al., 2006, 2007). Neurons within the $\mathrm{SNr}$ were visualized with infrared differential interference videomicroscopy using a X60 water-immersion objective. For voltage-clamp recordings micropipettes 2-5 $\mathrm{M} \Omega$ resistance were filled with internal saline containing high $\mathrm{Cl}^{-}$(in millimolar): 70 $\mathrm{KH}_{2} \mathrm{PO}_{4}, 36 \mathrm{KCl}, 2 \mathrm{MgCl}_{2}, 10$ HEPES, 1.1 EGTA, $0.2 \mathrm{Na}_{2} \mathrm{ATP}, 0.2$ $\mathrm{Na}_{3}$ GTP, 5 mM QX-314, 5 mM CsCl, and 0.1\% biocytin ( $\mathrm{pH} 7.2 ; 275$ $\mathrm{mOsM} / \mathrm{l}$ ) that allowed to record inward IPSCs from SNr neurons after field stimulation in the internal capsule (IC) $0.5-1.0 \mathrm{~mm}$ outside the SNr border (Radnikow and Misgeld, 1998; Wallmichrath and Szabo, 2002). Bipolar pencil shaped concentric tungsten electrodes, $11.5 \mu \mathrm{m}$ at the tip, and $1 \mathrm{k} \Omega \mathrm{DC}$ resistance were used. For current-clamp recordings we used internal saline of the following composition (in millimolar): $120 \mathrm{KSO}_{3} \mathrm{CH}_{4}, 10 \mathrm{NaCl}, 10$ EGTA, 10 HEPES, $1 \mathrm{CaCl}_{2}, 2 \mathrm{MgCl}_{2}$, 2 ATP-Mg, 0.3 GTP-Na (pH 7.3, 290 $\mathrm{mOsM} / \mathrm{l}$. Superfusion saline contained antagonists for glutamatergic receptors: $10 \mu \mathrm{M}$ 6-cyano-7-nitroquinoxaline-2,3-dione (CNQX) and $50 \mu \mathrm{M}$ D-(-)-2-amino-5-phosphopentanoic acid (APV) to isolate IPSCs. In parasagittal slices, 3 out of 10 recordings evoked pallidonigral IPSCs and the rest evoked striatonigral IPSCs (Figure 1). In parahorizontal slices 6 out of 10 recordings evoked pallidonigral IPSCs and the rest evoked striatonigral IPSCs. IPSCs from each source were easily discernible with electrophysiological techniques (Figure 1; Connelly et al., 2010) so that when an obvious mixture of IPSCs from both sources was obtained, it was discarded from the present analysis. Holding potential was $-80 \mathrm{mV}$. A paired-pulse protocol was employed with inter-pulse intervals of $50 \mathrm{~ms}$ to evaluate changes in the paired-pulse ratio (PPR) of evoked IPSCs (PPR $=2$ nd IPSC/1st IPSC) to verify presynaptic actions of transmitters (Ibáñez-Sandoval et al., 2006). Amplitude of first IPSC was used to build time courses of dopaminergic actions. Because striatonigral fibers pass through the $\mathrm{GPe}, \mathrm{D}_{2}$-class selective agonists were tested in slices taken from animals with a stereotaxic lesion (ibotenic acid) of the GPe (1.4 mm AP, 3.4 L, and $4.7 \mathrm{~mm} \mathrm{~V})$ and compared to recordings obtained without a lesion. The lesion further confirmed the differences of IPSCs from both sources. Ibotenic acid solution (dissolved in PBS adjusted to $\mathrm{pH} 7.4$ with $\mathrm{NaOH}$ $3.0 \mu \mathrm{g} / 0.4 \mu \mathrm{l}$ ) was used to lesion the GPe. These values closely followed Paxinos and Watson (1982) coordinates system.

Drugs were stored as dry aliquots and stock solutions were prepared prior to each experiment and added to the perfusion solution in the final concentration indicated. SKF 81297: (士)-6-Chloro-2,3,4,5-tetrahydro-1-phenyl-1H-3-benzazepine hydrobromide (10 nM-50 $\mu \mathrm{M}$ ); SCH 23390: 7-chloro-8-hydroxy3-methyl-1-phenyl-2,3,4,5-tetrahydro-1H-3-benzazepine (50 nM and $1 \mu \mathrm{M})$; CNQX: 6-cyano-7-nitroquinoxaline-2,3-dione $(10 \mu \mathrm{M})$, D-AP-5 :D-(-)-2-amino-5-phosphopentanoic acid ( $50 \mu \mathrm{M})$, bicuculline methiodide or methchloride $(10 \mu \mathrm{M})$, and QX-314 (5 mM inside the recording pipette), all were obtained from Sigma-Aldrich (St. Louis MO, USA). The neurons in the present work were $\mathrm{SNr}$ projection neurons ( $n=250$; see: Ibáñez-Sandoval et al., 2006). IPSCs parameters from different afferents, i.e., striatonigral and pallidonigral were compared with Mann-Whitney's U-tests. IPSCs parameters in the same synapses before and after adding a given drug were compared with Wilcoxon's $t$-tests. At least $10 \mathrm{~min}$ of stable recordings before and after administering the drugs were used to reach stable average IPSCs amplitudes. Each symbol represents mean and SEM of quantal variation in a single representative experiment. Sample averages are given in the text. Differences in parameters of fitted functions were compared by using their estimation error and Student's $t$-tests.

\section{RESULTS}

Figure 1 shows that striatonigral and pallidonigral synaptic inputs cannot be confused (Connelly et al., 2010). For similar stimulus, evoked striatonigral IPSCs were significantly smaller than pallidonigral IPSCs (mean \pm SEM for the first IPSC of a paired response): $235 \pm 50 \mathrm{pA}(n=32)$ vs. $1399 \pm 152 \mathrm{pA}(n=20 ; P<0.0001)$ respectively. Responses to paired-pulse stimulation were also different: striatonigral IPSCs showed paired-pulse facilitation: $\mathrm{PPR}=1.41 \pm 0.07$ (Figures 1A,G; $n=30$ ) whereas pallidonigral IPSCs always exhibited paired-pulse depression: $\mathrm{PPR}=0.4 \pm 0.06$ (Figures 1B,G; $n=19 ; P<0.0001$ ), although not always as strong as that depicted in Figure 1D. Short-term plasticity (10 pulses at $20 \mathrm{~Hz}$ ) exhibited facilitation without failures in striatonigral connections (Figure 1C; $n=8$ ), whereas it exhibited depression with intermittent failures in pallidonigral synapses (Figure 1D; $n=4$ ). The decay time constant/rise time ratio was also different: $4.5 \pm 0.5$ $(n=30)$ in striatonigral synapses and $12.6 \pm 0.8$ in pallidonigral synapses $(n=19 ; P<0.0001)$ showing that pallidonigral IPSCs are briefer than striatonigral ones (Connelly et al., 2010). IntensityAmplitude (I-A) relationships were also constructed and fitted to a three parameter sigmoidal function (Tecuapetla et al., 2005):

$A(I)=\frac{A_{\max }}{1+e^{-k\left(I-I_{h}\right)}}$ 
A

C<smiles>[AlH2]</smiles>

trrnnrur

E

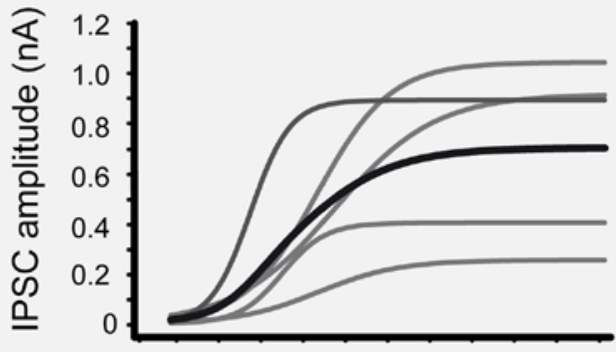

B

pallidonigral

$100 \mathrm{pA} \bigsqcup_{50 \mathrm{~ms}}^{500 \mathrm{pA}}$

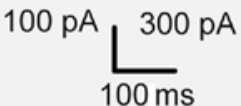

D

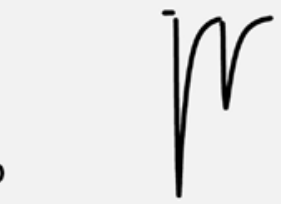

$\begin{array}{lllllllllll}0 & 1 & 2 & 3 & 4 & 5 & 6 & 7 & 8 & 9 & 10\end{array}$

F

\section{$X$ threshold units}

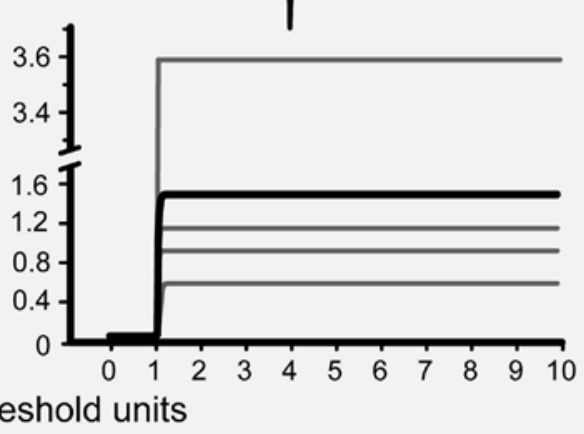

G

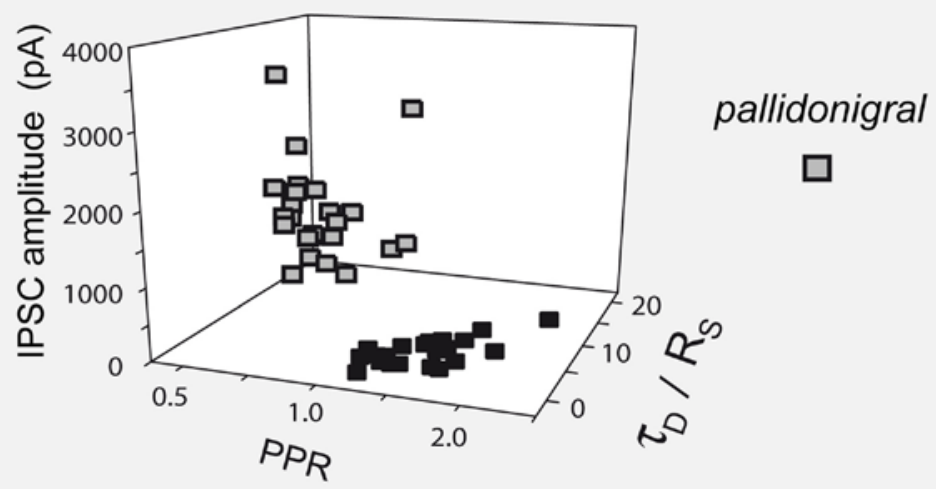

FIGURE 1 | Differences between striatonigral and pallidonigral inhibitory postsynaptic currents. (A) Striatonigral inhibitory postsynaptic currents (IPSCs) exhibited paired-pulse facilitation. (B) Pallidonigral IPSCs exhibit paired-pulse depression. (C) Short-term synaptic plasticity (STP) from striatonigral afferents is facilitation. (D) STP from pallidonigral afferents is depression with numerous failures. (E) Intensity-amplitude plots (I-A plots) from striatonigral IPSCs exhibit a sigmoidal shape. (F) I-A plots from pallidonigral IPSCs exhibit a jump to maximal amplitude after reaching threshold. I-A plots were fitted to: $A(I)=A_{\max } /\left(1+e^{-k(I-l)}\right)$ where $A(I)=I P S C$ amplitude as a function of stimulus intensity, $A_{\max }=$ maximal amplitude reached, $k=$ slope factor, and $I_{h}=$ stimulus intensity necessary to reach IPSC amplitude equal to half maximal amplitude. All parameters were significantly different. (G) Cluster plot showing that IPSCs from these sources can be separated. PPR $=$ paired-pulse ratio. $\tau_{D}=$ decay time constant of IPSCs. $R_{\mathrm{S}}=$ rise time of IPSCs. where $A(I)=$ IPSC amplitude as a function of stimulus intensity, $A_{\max }=$ maximal amplitude reached, $k=$ slope factor, and $I_{h}=$ stimulus intensity necessary to reach IPSC amplitude equal to half maximal amplitude. All three parameters were significantly different when comparing IPSCs from striatonigral vs. pallidonigral afferents: $A_{\max }$ : $430 \pm 3$ pA vs. $1512 \pm 10$ pA $(n=8 ; P<0.0001) ; k=3.6 \pm 0.3$ vs. $14 \pm 5$ $(n=8 ; P<0.0001)$ and $I_{h}: 2.3 \pm 0.2$ vs. $1.1 \pm 0.1(n=8 ; P<0.005)$.

These features coincide with a previous report (Connelly et al., 2010) and were verified qualitatively by evoking IPSCs from either the subthalamic nucleus (NST) or the GPe, however, in these occasions IPSCs were considerably smaller and amplitude could not be compared by itself given the different distances from stimulating and recording electrodes (not shown). Striatonigral IPSCs features have been well described (Radnikow and Misgeld, 1998; Wallmichrath and Szabo, 2002; Beurrier et al., 2006; Chuhma et al., 2011) as well as IPSCs from pallidal origin making synapses into the SNr and other nuclei (Tecuapetla et al., 2005; Baufreton et al., 2009; Connelly et al., 2010). Finally, three of these variables were used to build a cluster plot (Figure 1G) which confirmed the easiness to separate IPSCs from each source. 
The actions of selective dopamine receptor agonists for $\mathrm{D}_{1}$ - and $\mathrm{D}_{2}$-receptor classes were tested. As it has been repeatedly demonstrated, the action of dopaminergic $\mathrm{D}_{1}$-class selective agonists at nanomolar concentrations was that of enhancing striatonigral IPSCs (Floran et al., 1990; Radnikow and Misgeld, 1998; Chuhma et al., 2011): striatonigral IPSC increased $153 \pm 10 \%$ after $300 \mathrm{nM} \mathrm{SKF}$ $81297(n=15 ; P<0.001)$ and the paired-pulse ratio (PPR = IPSC2/ IPSC1) decreased from $1.4 \pm 0.13$ in the control to $1.0 \pm 0.12$ during SKF $81297(P<0.001)$, confirming a presynaptic modulation. These actions were reversible and blocked by $100 \mathrm{nM}$ of the $\mathrm{D}_{1}$-antagonist SCH 23390 ( $n=5$; not shown here but see below) indicating that at these concentrations the action is specific. In addition, here we show that the agonists have no significant action on pallidonigral IPSCs (cf., Figures 2A,B).

Contrasting results were obtained when a selective $\mathrm{D}_{2}$-class receptor agonist, $500 \mathrm{nM}$ quinelorane, was employed: striatonigral IPSCs suffered no significant alteration (Figure 2C; $n=5 \mathrm{NS}$; Chuhma et al., 2011) whereas pallidonigral IPSCs were significantly inhibited (Figure 2D) by $73 \pm 18 \%$ (Figure 2D; $n=19 ; P<0.0001$ ). PPR increased from $0.76 \pm 0.1$ to $1.83 \pm 0.2(P<0.001)$, suggesting a presynaptic action. These actions were reversible and blocked by $500 \mathrm{nM}$ sulpiride, $\mathrm{a}_{2}$-antagonist ( $n=5$; not shown but see below).

Summarizing, physiological action of $\mathrm{D}_{1}$-receptor agonists on striatonigral terminals is that of IPSC enhancement as previously shown (Floran et al., 1990; Radnikow and Misgeld, 1998; Chuhma et al., 2011) with no action on pallidonigral terminals. On the other hand, physiological action of $\mathrm{D}_{2}$-receptor agonists on pallidonigral IPSCs is that of depression with no significant action on striatonigral terminals.

Therefore we were forced to infer that reports about $\mathrm{D}_{1}$-mediated inhibition of striatonigral terminals (Miyazaki and Lacey, 1998) were either involving a non-specific action, a pallidal contamination, or both. To test this hypothesis we used larger micromolar concentrations of the $\mathrm{D}_{1}$-agonist while evoking IPSCs from both pathways. Figures 3 A,B show that $5 \mu \mathrm{M}$ SKF 81297 decreased IPSCs evoked from both set of terminals. Striatonigral IPSC decreased $82 \pm 13 \%(n=18 ; P<0.001)$ and pallidonigral responses decreased by $35 \pm 15 \%(n=6 ; P<0.005)$. These actions could not be blocked by micromolar concentrations of SCH 23390 (not shown), suggesting that they were not specific. In view of these results we built a concentration-response relationship (C-R plot) using a wide range of SKF 81297 concentrations while stimulating striatonigral afferents. This $\mathrm{C}-\mathrm{R}$ plot can be seen in Figure 3C: it is biphasic. When the Hill equation was fitted to the ascending (specific part) $\mathrm{EC}_{50}$ was $440 \pm 60 \mathrm{nM}$ and the Hill coefficient $1.6 \pm 0.2$, suggesting cooperativity and a specific action at submicromolar concentrations. Moreover, the fact that pallidonigral inputs are also affected when they do not respond when submicromolar concentrations of agonists are used confirmed non-specific actions.

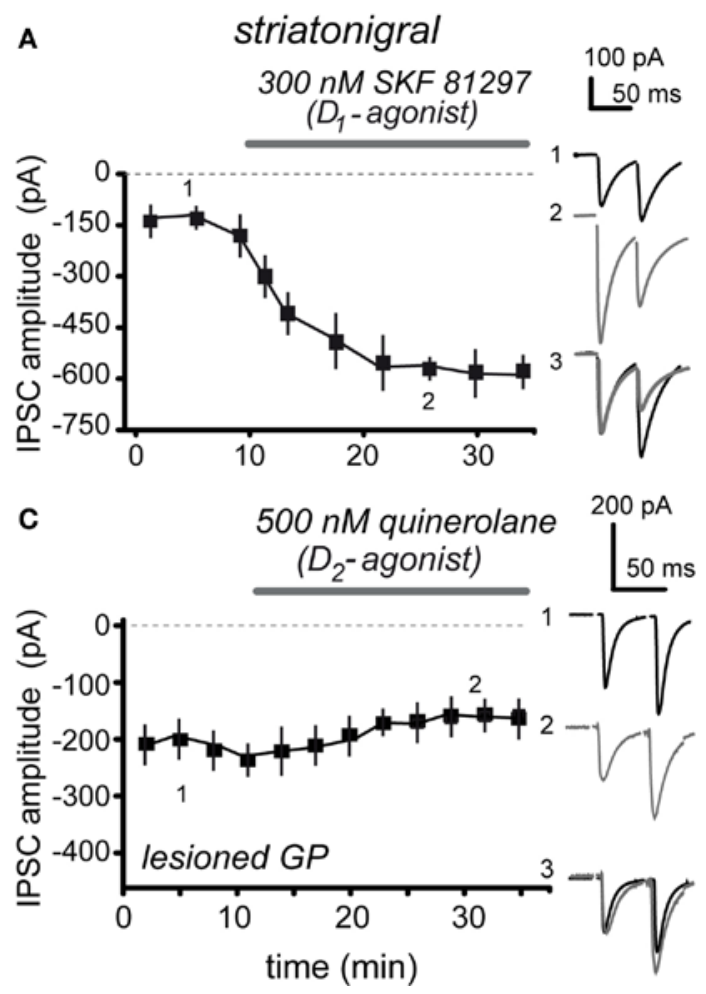

FIGURE 2 | Contrasting actions of $D_{1}$ - and $D_{2}$-receptor agonists on striatonigral and pallidonigral IPSCs. (A) $100 \mathrm{nM}$ of the selective $\mathrm{D}_{1}$-class receptor agonist, SKF 81297, enhanced striatonigral IPSCs. (B) 100 nM SKF 81297, had no action on pallidonigral IPSCs. (C) $500 \mathrm{nM}$ of the selective $\mathrm{D}_{2}$-class receptor agonist, quinelorane, had no significant action on striatonigral IPSCs. In

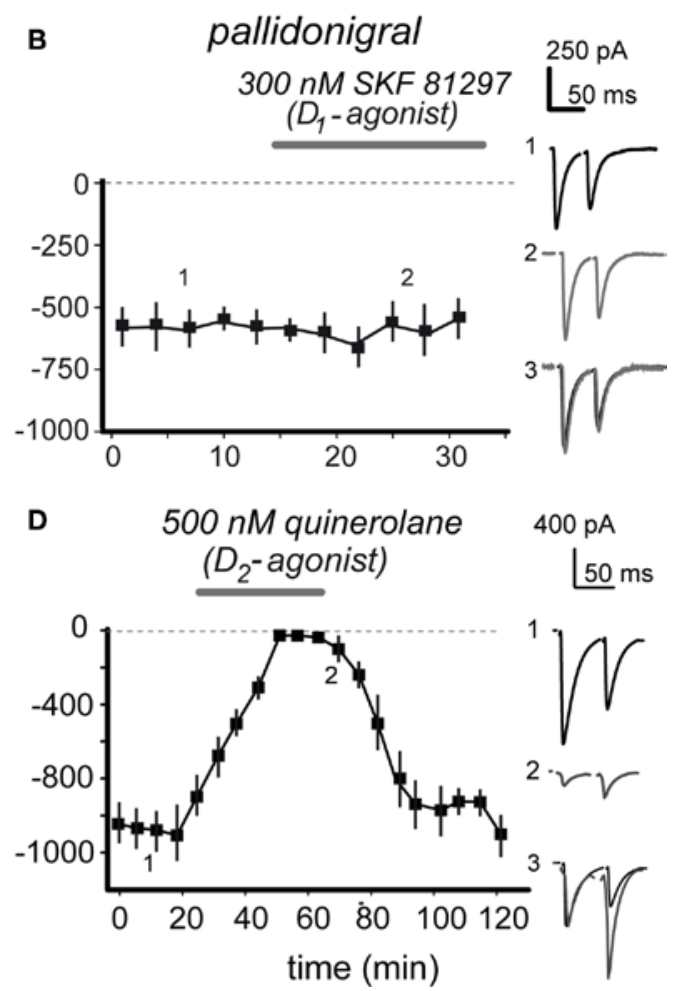

some cases the GPe was lesioned to better avoid contamination from pallidonigral afferents. (D) $500 \mathrm{nM}$ quinelorane greatly reduced pallidonigral IPSCs. Note changes in PPR accompanying significant effects. Record 3 in each frame is the superimposition of records 1 and 2 after normalization of the first IPSC to better appreciate the PPR change when it is present. 

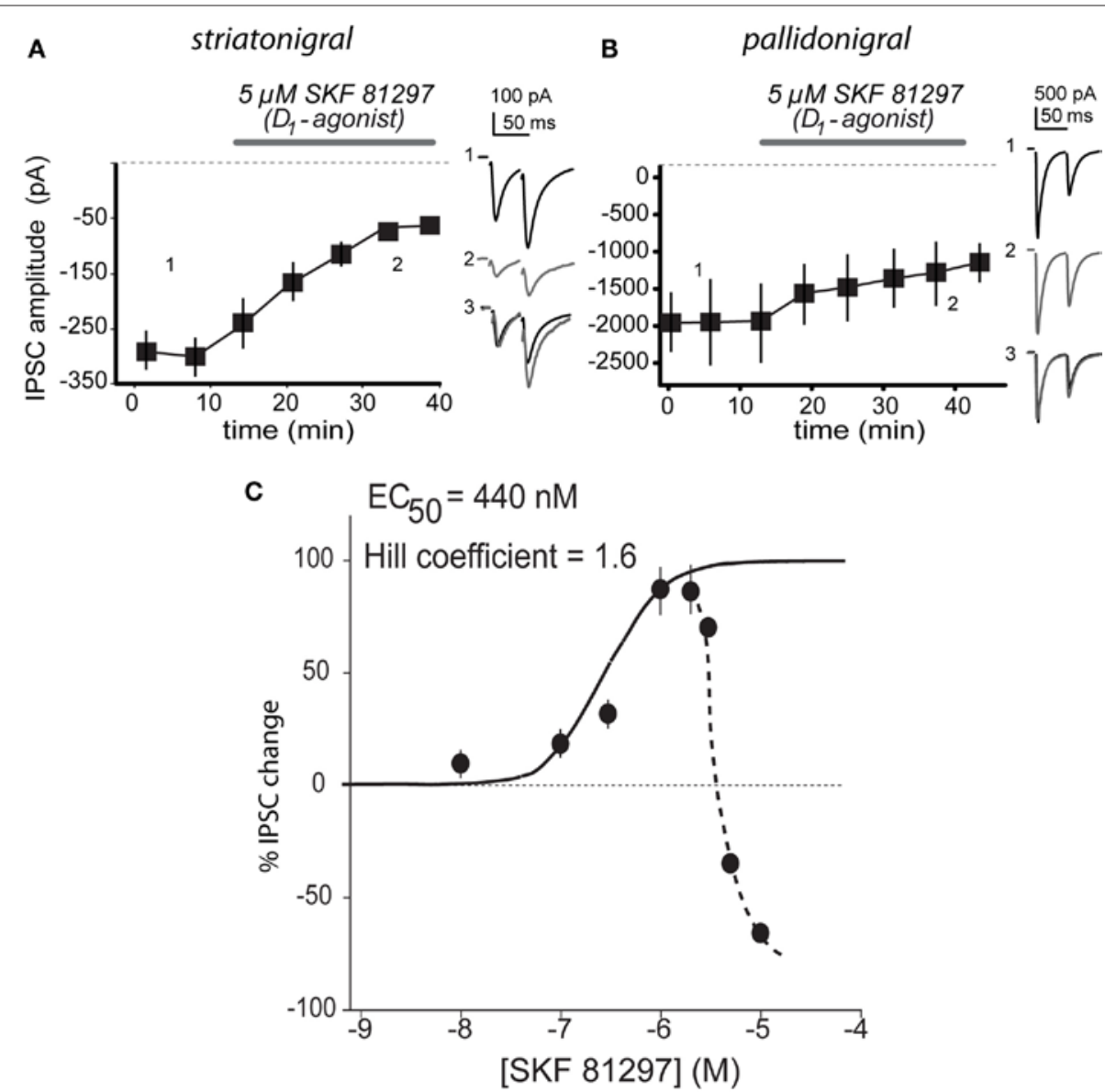

FIGURE 3 | Biphasic concentration-response relationship of $D_{1}$-agonist. (A) $5 \mu \mathrm{M}$ SKF 81297 decreased IPSCs evoked from striatonigral terminals. (B) $5 \mu \mathrm{M}$ SKF 81297 decreased IPSCs evoked from pallidonigral terminals. Record 3 in each frame is the superimposition of representative records 1 and 2 after normalization of the first IPSC to better appreciate the PPR change when it is present. (C) Concentrationresponse relationship ( $\mathrm{C}-\mathrm{R}$ plot) showed a biphasic response on striatonigral responses: once $3 \mu \mathrm{M}$ are surpassed, IPSCs decay with increasing concentrations. Ascending $\mathrm{C}-\mathrm{R}$ plot was fitted with the Hill equation finding a submicromolar $\mathrm{EC}_{50}$.
Given the low concentrations of agonists needed to activate $\mathrm{D}_{1}$ - and $\mathrm{D}_{2}$-class receptors in their respective terminals (striatonigral and pallidonigral) we inferred that, perhaps, endogenous extracellular dopamine exerts a tonic action on these receptors. Figure 4 shows that this hypothesis is correct. Figures 4 A,B show that $50 \mathrm{nM}$ of a $\mathrm{D}_{1}$-class receptor selective antagonist, $\mathrm{SCH} 23390$, are enough to inhibit striatonigral IPSCs with no significative action on pallidonigral IPSC. Striatonigral IPSC decreased from $319 \pm 75$ in the control to $150 \pm 11 \mathrm{pA}$ after $50 \mathrm{nM} \mathrm{SCH} 23390(n=12$; $P<0.02)$. Figures 4C,D confirm these findings and further show that potency and speediness of $\mathrm{D}_{1}$-action is concentration dependent. Striatonigral IPSC is greatly reduced - almost abolished - when a low micromolar antagonist concentration is maintained in the superfusion (Figure 4C). The effect is reversible (not shown). Traces chosen at different times during the time course, superimposed, and normalized to the amplitude of the first IPSC, show that the PPR is greatly increased from $1.9 \pm 0.4$ in the control to $2.4 \pm 0.6$ after SCH $23390(n=21 ; P<0.001$; when the IPSC is abolished PPR cannot be measured); confirming a presynaptic site of action. On the other hand, SCH 23390 did not produce any action on pallidonigral IPSC at any concentration (Figure 4D). In summary,
$\mathrm{D}_{1}$-receptors in direct pathway striatonigral terminals are sensitive detectors of extracellular dopamine. Moreover, blockade of dopaminergic action can reduce striatonigral synaptic reliability to a minimum.

In addition, Figures 4E,F illustrate the actions of selective antagonists for $\mathrm{D}_{3}$ - and $\mathrm{D}_{4}$-type dopamine receptors: $500 \mathrm{nM}$ U-9914A, a selective $D_{3}$-type receptor antagonist enhanced pallidonigral IPSCs by $262 \pm 16 \%$ after $(n=12 ; P<0.001)$ while the PPR decreased from $0.87 \pm 0.08$ in the control to $0.51 \pm 0.1$ during the antagonist. On the other hand, $500 \mathrm{nM}$ LY750, a selective $\mathrm{D}_{4}$-type receptor antagonist increased pallidonigral IPSCs by $170 \pm 20 \%(n=7 ; P<0.001)$ while PPR decreased from $0.78 \pm 0.07$ in the control to $0.57 \pm 0.09$ during the blockade. Sulpiride a generic $\mathrm{D}_{2}$-class receptor antagonist had similar actions (not shown): pallidonigral IPSCs increased by $150 \pm 14 \%(n=3)$ while PPR decreased from $0.77 \pm 0.03$ in the control to $0.35 \pm 0.01$ during blockade. To summarize, $\mathrm{D}_{3 / 4}$-receptors in pallidonigral terminals (Murray et al., 1994; Bevan et al., 1996; Marshall et al., 2001; Rivera et al., 2003; Seeman et al., 2006; Acosta-García et al., 2009; Gasca-Martinez et al., 2010) are sensitive to extracellular dopamine, which has the role of tonically repressing the force 

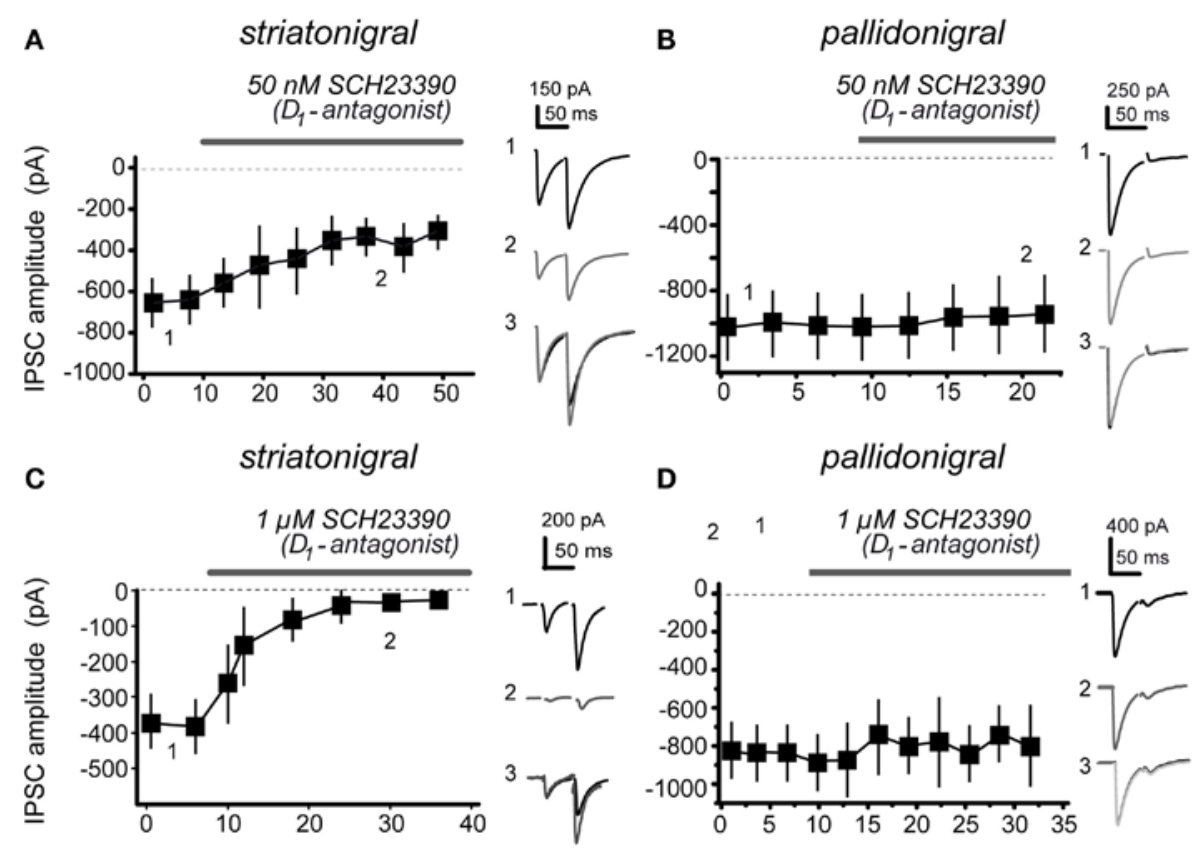

D
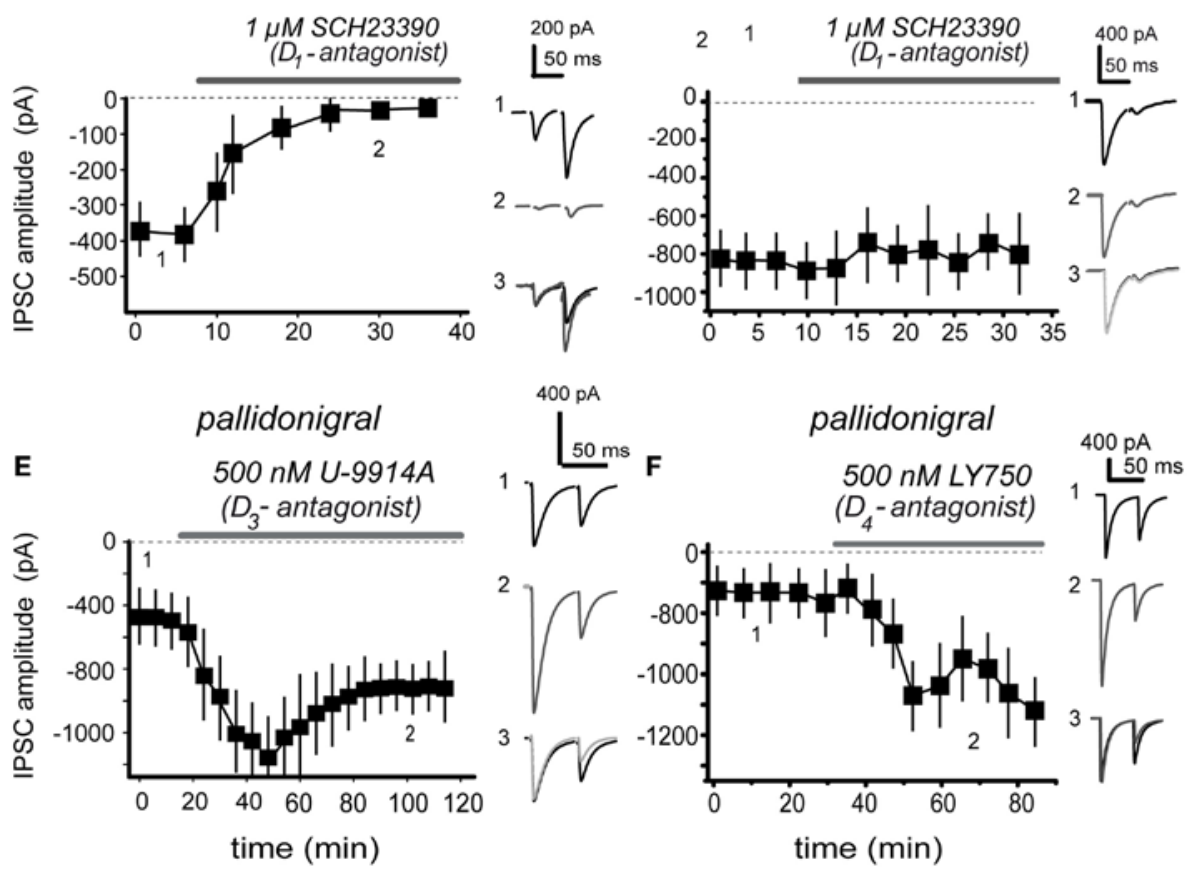

FIGURE 4 | Presynaptic dopamine receptors sense endogenous extracellular dopamine. (A) $50 \mathrm{nM}$ of selective dopamine $\mathrm{D}_{1}$-receptor antagonist, SCH 3390, are enough to reduce striatonigral IPSC amplitude. (B) The same antagonist concentration has no action on pallidonigral IPSC. (C) Increasing $D_{1}$-antagonist concentration $(1 \mu \mathrm{M})$ greatly reduced IPSC while speeding up dopamine actions on striatonigral IPSC, suggesting a concentration dependent mechanism. (D) The same antagonist concentration has no action on pallidonigral IPSC. (E) 500 nM U-9914A, a selective $D_{3}$-type receptor antagonist, significantly enhanced pallidonigral IPSC. Note a partial reversion. (F) 500 nM LY750, a selective $D_{4}$-type receptor antagonist, significantly increased pallidonigral IPSC. Record 3 in each frame is the superimposition of representative records 1 and 2 after normalization of the first IPSC to better appreciate the PPR change when it is present. of these synapses. Some functional differences in the actions of these receptor types perhaps deserve further investigation (cf., Figures 4E,F).

Finally, because subthalamonigral terminals are also tonically controlled by presynaptic dopamine receptors $\left(\mathrm{D}_{1}\right.$ - and $\mathrm{D}_{2}$-class; IbáñezSandoval et al., 2006) and because blockade of these receptors enhance subthalamonigral EPSCs (Ibáñez-Sandoval et al., 2006), we propose the following hypothesis based in the present and previous results (Radnikow and Misgeld, 1998; Acosta-García et al., 2009; Chuhma et al., 2011): that acute blockade of both $\mathrm{D}_{1}$ - and $\mathrm{D}_{2}$-class (including $\mathrm{D}_{3 / 4}$-types) receptors (by $1 \mu \mathrm{M}$ SCH 23390 plus $1 \mu \mathrm{M}$ sulpiride) altogether may decrease direct pathway synapses (striatonigral) and, at the same time, enhance indirect pathway synapses (subthalamonigral and pallidonigral), both actions being required in physiopathological models of Parkinsonism to elicit the signs of the disease. The result can be seen in Figure 5: SNr neurons shifted from a tonic firing pattern in the control to a bursting firing pattern after blockade of dopamine receptors ( $n=7$ neurons; Ibáñez-Sandoval et al., 2007).

One of the most supported neuronal correlates of Parkinsonism: recurrent bursting in $\mathrm{SNr}$ neurons, was similar to that previously reported in vivo and in vitro (Hammond et al., 2007; IbáñezSandoval et al., 2007; Walters et al., 2007; Walters and Bergstrom, 2009; Zold et al., 2009). Transitions from tonic to bursting firing mode can rarely be seen spontaneously in control preparations (Ibáñez-Sandoval et al., 2007).

To see whether excitatory subthalamonigral afferents, that is, the STN-GP circuit, was participating in this firing behavior, we added the NMDA-receptor antagonist APV $(50 \mu \mathrm{M})$ to provoke a partial 

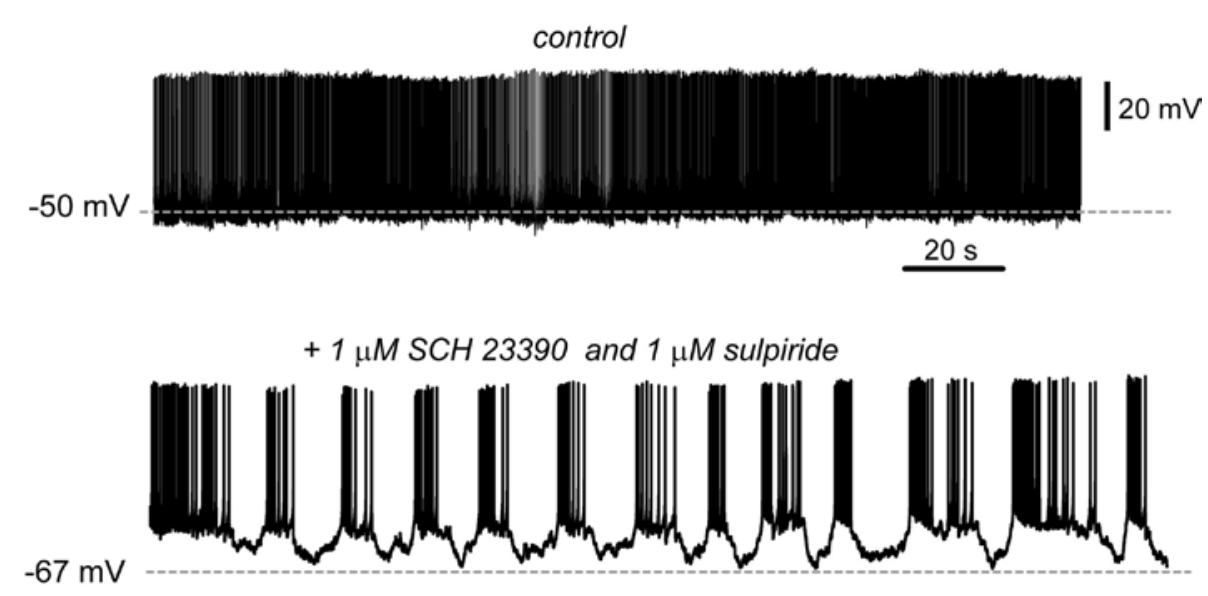

$+1 \mu \mathrm{M} \mathrm{SCH} 23390$ and $1 \mu \mathrm{M}$ sulpiride

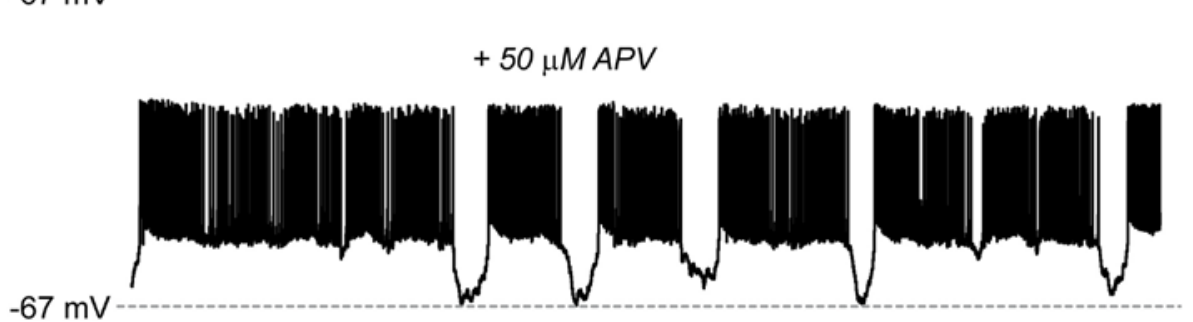

FIGURE 5 | Blockade of dopamine receptors changes the firing pattern of SNr neurons. Spontaneous tonic firing was the usual firing pattern recorded in $\mathrm{SNr}$ neurons in control conditions (top). During acute blockade of dopamine $D_{1}$ and $\mathrm{D}_{2}$-class receptors ( $1 \mu \mathrm{M} \mathrm{SCH} 23390$ plus $1 \mu \mathrm{M}$ sulpiride) SNr firing pattern shifted from tonic to a bursting pattern in $n=7$ cells; two cells exhibited bursting before adding the blockers. To see whether besides striatonigral and pallidonigral afferents, subthalamonigral afferents were also contributing to this firing mode, the glutamate NMDA-receptor antagonist, $50 \mu \mathrm{M}$ APV was added to the bath saline (bottom). The firing tended to return to a tonic firing pattern but it was frequently interrupted by sudden hyperpolarizations. block of STN influence. The result was that the firing pattern became less bursty and more tonic (Ibáñez-Sandoval et al., 2007), however, firing was still abruptly interrupted by sudden hyperpolarizations, probably coming from enhanced pallidonigral inputs.

\section{DISCUSSION}

The present work shows that extracellular dopamine concentrations are tonically being sensed by the synaptic terminals of inhibitory inputs to the $\mathrm{SNr}$ in opposite ways. Thus, blockade of $\mathrm{D}_{1}$-type receptors in striatonigral (direct pathway) afferents decreased striatonigral inhibition while blockade of $\mathrm{D}_{3 / 4}$-types receptors in pallidonigral terminals enhanced pallidonigral inhibition. Pallidonigral afferents are presynaptically controlled by $\mathrm{D}_{2}$-class but not $\mathrm{D}_{1}$-class receptors. Still other inputs to the $\mathrm{SNr}$ have to be studied to see whether they are presynaptically modulated.

\section{STRIATONIGRAL AND PALLIDONIGRAL IPSCs ARE DIFFERENT}

Striatonigral IPSCs are smaller but last longer than pallidonigral IPSCs. In addition, they exhibit short-term facilitation and are positively regulated by $\mathrm{D}_{1}$-receptors (Floran et al., 1990; Radnikow and Misgeld, 1998; Connelly et al., 2010). Strong evidence for the last property has been obtained with optogenetic techniques (Chuhma et al., 2011), supporting the present and previous reports. In comparison, pallidonigral IPSCs are larger and exhibit different degrees of short-term depression (Connelly et al., 2010). The two classes of inhibition are so different that cannot be confused. Pallidonigral IPSCs are negatively regulated by $\mathrm{D}_{2}$-class receptors and have no
$\mathrm{D}_{1}$-modulation. Notably, short-term facilitation works as a high pass filter of incoming inputs whereas short-term depression works as a low pass filter (Abbott and Regehr, 2004). In contrast, subthalamonigral excitatory synapses are controlled by both $\mathrm{D}_{1}-$ and $\mathrm{D}_{2}$-class receptors (Ibáñez-Sandoval et al., 2006).

Selective dopamine receptor agonists exert their specific actions at nanomolar or low micromolar concentrations but higher concentrations become non-specific, thus explaining previous contradictions (cf., Miyazaki and Lacey, 1998; Radnikow and Misgeld, 1998) and probably similar discrepancies within the striatal/accumbinal circuitry (Guzman et al., 2003; Taverna et al., 2005). To summarize: $\mathrm{D}_{1}$-receptor activation enhances GABA release in terminals from medium spiny neurons of the direct pathway whereas $\mathrm{D}_{3 / 4}$-receptor activation represses GABA release from pallidonigral terminals.

\section{STRIATONIGRAL AND PALLIDONIGRAL DOPAMINE RECEPTORS SENSE EXTRACELLULAR AMBIENT ENDOGENOUS DOPAMINE}

Because nanomolar concentrations of selective receptor agonist are needed to modify transmission, we hypothesized that administrations of selective dopamine receptor antagonist would disclose the actions of endogenous dopamine present in the extracellular space. Therefore, to disclose the action of ambient endogenous dopamine we applied selective $\mathrm{D}_{1}$ - and $\mathrm{D}_{3 / 4}$-receptor antagonists. Our findings were that according to the concentration, suppression of endogenous dopamine action greatly reduced striatonigral transmission while it enhanced pallidonigral transmission. These results indicated that extracellular dopamine concentrations are 
regulating synaptic release probability in both types of connections, increasing release probability in striatonigral synapses and decreasing release probability in pallidonigral synapses.

Therefore, the following working hypothesis resulted from the present experiments: tonic levels of dopamine in $\mathrm{SNr}$ are necessary to maintain the normal function of direct pathway connections and maintain in check indirect pathway synapses from both pallidonigral terminals (present work) and subthalamonigral terminals (IbáñezSandoval et al., 2007). The logical functional consequence of this hypothesis was tested: blockade of tonic dopamine action would enhance indirect pathway transmission and reduce direct pathway input thus yielding a previously observed neuronal correlate of Parkinsonism: SNr neurons shifted their tonic firing pattern to a bursting firing pattern typical of subjects with the disease (Takakusaki et al., 2004; Rivlin-Etzion et al., 2006; Hammond et al., 2007; IbáñezSandoval et al., 2007; Walters et al., 2007; Zold et al., 2009).

Tonic spontaneous firing is preserved in the slice preparation in both GPe and STN (e.g., Beurrier et al., 1999; Chan et al., 2011). Calcium imaging techniques recording dozens of cells simultaneously within the striatal circuit show that there is always some spontaneous activity in striatal spiny neurons in control conditions (CarrilloReid et al., 2008). Moreover, there is a great amount of convergence between the striatum and the substantia nigra and inhibitory striatonigral events are of large amplitude (e.g., Chuhma et al., 2011). Thus, for a SNr neuron there always may be some striatal cell firing. Therefore, the lost balance between direct and indirect pathways by the acute blockade of dopamine receptors is the most probable cause of bursting in $\mathrm{SNr}$ neurons during the present experiments.

Postsynaptic dopamine receptors on $\mathrm{SNr}$ neurons cannot explain bursting because their function is to increase tonic firing frequency via a cation current; their blockade resulting in lower tonic frequency with irregularities, but not continuous bursting behavior (Lee and Tepper, 2007; Zhou et al., 2009).

\section{FUNCTIONAL CONSEOUENCES}

Because the change in firing pattern of $\mathrm{SNr}$ neurons was achieved acutely by blocking dopamine receptors one can speculate what would happen during a chronic absence of dopamine in the output

\section{REFERENCES}

Abbott, L. F., and Regehr, W. G. (2004). Synaptic computation. Nature 431,796-803.

Acosta-García, J., Hernández-Chan, N., Paz-Bermúdez, F., Sierra, A., Erlij, D., Aceves, J., and Florán, B. (2009). D4 and D1 dopamine receptors modulate $[3 \mathrm{H}]$ GABA release in the substantia nigra pars reticulata of the rat. Neuropharmacology 57, 725-730.

Albin, R. L., Young, A. B., and Penney, J. B. (1989). The functional anatomy of basal ganglia disorders. Trends Neurosci. 12, 366-375.

Alexander, G. E., and Crutcher, M. D. (1990). Functional architecture of basal ganglia circuits: neural substrates of parallel processing. Trends Neurosci. 13, 266-271.
Altar, C. A., and Hauser, K. (1987). Topography of substantia nigra innervation by $\mathrm{D} 1$ receptor-containing striatal neurons. Brain Res. 410, 1-11.

Avila, I., Parr-Brownlie, L. C., Brazhnik, E., Castaneda, E., Bergstrom, D. A., and Walters, J. R. (2010). Beta frequency synchronization in basal ganglia output during rest and walk in a hemiparkinsonian rat. Exp. Neurol. 221, 307-319.

Bateup, H. S., Santini, E., Shen, W., Birnbaum, S., Valjent, E., Surmeier, D. J., Fisone, G., Nestler, E. J., and Greengard, P. (2010). Distinct subclasses of medium spiny neurons differentially regulate striatal motor behaviors. Proc. Natl. Acad. Sci. U.S.A.

Baufreton, J., Kirkham, E., Atherton, J. F., Menard,A., Magill,P.J., Bolam,J.P., and 107, 14845-14850.

nuclei of the BG. The absence of dopamine (e.g., Parkinsonism) may reduce the function of direct pathway synapses in such a way that maintaining this state of affairs in the long-time would lead to loss of direct pathway synapses due to long-term synaptic plasticity. In contrast, maintaining a high function in pallidonigral and subthalamonigral synapses would produce long-term potentiation of these synapses. These events taken together may tend to change the circuitry permanently, making not only L-DOPA inefficient but, perhaps, making the chronic diseased circuitry radically different than the control or healthy circuit: that is, more dependent on the subthalamopallidal loop (Magill et al., 2001; Baufreton et al., 2009).

Bursting in SNr neurons leads to tremor and rigidity (Hemsley and Crocker, 1998; Takakusaki et al., 2004). Moreover, this configuration of synaptic weights would lead to the loss of high pass filtering of SNr inputs (direct pathway's short-term facilitation) and an increase in low pass filtering of SNr inputs (pallidonigral short-term depression; Abbott and Regehr, 2004) setting the stage to favor the entrance to an akinetic frequency lock (Hutchison et al., 2004; Avila et al., 2010). Consequently, therapeutic ways of activating the direct pathway in the chronic patient may be fundamental to avoid irreversible plastic changes in the network (Hammond et al., 2007; Walters et al., 2007; Walters and Bergstrom, 2009; Zold et al., 2009; Bateup et al., 2010; Kravitz et al., 2010).

\section{ACKNOWLEDGMETS}

We thank A. Laville, D. Tapia and G. X. Ayala for helpful comments and technical support. This work was funded by Investigacion Multidisciplinaria de Proyectos Universitarios de Liderazgo y Superacion Academica (IMPULSA)-Universidad Nacional Autónoma de México (UNAM) 03, Dirección General de Asuntos del Personal Académico-UNAM Grants IN-205610 to José Bargas, and IN-206010 to Elvira Galarraga. Fundación Miguel Alemán AC to José Bargas. Pavel E. Rueda-Orozco was postdoctoral fellow at UNAM. Osvaldo Ibañez-Sandoval Ricardo Hernandez, José de Jesús Aceves, and Víctor Plata had or have CONACyT-Mexico doctoral fellowships.

Bevan,M.D. (2009). Sparse but selective and potent synaptic transmission from the globus pallidus to the subthalamic nucleus. J. Neurophysiol. 102, 532-545.

Beurrier, C., Ben-Ari, Y., and Hammond, C. (2006). Preservation of the direct and indirect pathways in an in vitro preparation of the mouse basal ganglia. Neuroscience 140, 77-86.

Beurrier, C., Congar, P., Bioulac, B., and Hammond, C. (1999). Subthalamic nucleus neurons switch from singlespike activity to burst-firing mode. J. Neurosci. 19, 599-609.

Bevan, M. D., Bolam, J. P., and Crossman, A. R. (1994). Convergent synaptic input from the neostriatum and the subthalamus onto identified nigrothalamic neurons in the rat. Eur. J. Neurosci. 16, 320-334.
Bevan, M. D., Smith, A. D., and Bolam, J. P. (1996). The substantia nigra as a site of synaptic integration of functionally diverse information arising from the ventral pallidum and the globus pallidus in the rat. Neuroscience 75, 5-12.

Carrillo-Reid, L., Tecuapetla, F., Tapia, D., Hernández-Cruz, A., Galarraga E., Drucker-Colin, R., and Bargas, J. (2008). Encoding network states by striatal cell assemblies. J. Neurophysiol. 99, 1435-1450.

Chan, C. S., Glajch, K. E., Gertler, T. S., Guzman,J.N., Mercer,J.N., Lewis,A.S., Goldberg, A. B., Tkatch, T., Shigemoto, R., Fleming, S. M., Chetkovich, D. M., Osten, P., Kita, H., and Surmeier, D. J. (2011). HCN channelopathy in external globus pallidus neurons in models 
of Parkinson's disease. Nat. Neurosci. 14, 85-92.

Chevalier, G., Vacher, S., Deniau, J. M., and Desban, M. (1985). Disinhibition as a basic process in the expression of striatal function. I. The striato-nigral influence on tecto-spinal/tectodiencephalic neurons. Brain Res. 334, 215-226.

Chuhma, N., Tanaka, K. F., Hen, R., and Rayport, S. (2011). Functional connectome of the striatal medium-spiny neuron. J. Neurosci. 31, 1183-1192.

Connelly, W. M., Schulz, J. M., Lees, G., and Reynolds, J.N. (2010). Differential short-term plasticity at convergent inhibitory synapses to the substantia nigra pars reticulata. J. Neurosci. 30, 14854-14861.

DeLong, M. R. (1990). Primate models of movement disorders of basal ganglia origin. Trends Neurosci. 13, 281-285.

Deniau, J. M., Menetrey, A., and Charpier, S. (1996). The lamellar organization of the rat substantia nigra pars reticulata: segregated patters of striatal afferents and relationship to the topography of corticostriatal projections. J. Neurosci. 73, 761-781.

Floran, B., Aceves, J., Sierra, A., and Martinez-Fong, D. (1990). Activation of D1 dopamine receptors stimulates the release of GABA in the basal ganglia of the rat. Neurosci. Lett. 116,136-140.

Gale, S. D., and Perkel, D. J. (2010). Anatomy of a songbird basal ganglia circuit essential for vocal learning and plasticity. J. Chem. Neuroanat. 39, 124-131.

Gasca-Martinez,D.,Hernandez,A., Sierra, A., Valdiosera, R., Anaya-Martinez, V., Floran, B., Erlij, D., and Aceves, J. (2010). Dopamine inhibits GABA transmission from the globus pallidus to the thalamic reticular nucleus via presynaptic D4 receptors. Neuroscience 169, 1672-1681.

Grillner, S., Markram, H., De Schutter, E., Silberberg, G., and LeBeau, F. E. (2005). Microcircuits in action from CPGs to neocortex. Trends Neurosci. 28, 525-533.

Grillner, S., Wallén, P., Saitoh, K., and Kozlov, A. B. (2008). Robertson neural bases of goal-directed locomotion in vertebrates an overview. Brain Res. Rev. 57, 2-12.

Grofová, I., and Rinvik, E. (1970). An experimental electron microscopic study on the striatonigral projection in the cat. Exp. Brain Res. 11, 249-262.

Guzman, J. N., Hernandez, A., Galarraga, E., Tapia, D., Laville, A., Vergara, R. Aceves, J., and Bargas, J. (2003). Dopaminergic modulation of axon collaterals interconnecting spiny neurons of the rat striatum. J. Neurosci. 23, 8931-8940.

Haber, S. N. (2003). The primate basal ganglia: parallel and integrative networks. J. Chem. Neuroanat. 26, 317-330.

Hammond, C., Bergman, H., and Brown, P. (2007). Pathological synchronization in Parkinson's disease: networks, models and treatments. Trends Neurosci. 30, 357-364.

Hemsley, K. M., and Crocker, A. D. (1998). The effects of an irreversible dopamine receptor antagonist, N-ethoxycarbonyl-2-ethoxy-1,2dihydroquinoline (EEDQ), on the regulation of muscle tone in the rat: the role of the substantia nigra. Neurosci. Lett. 251, 77-80.

Hikosaka, O., Takikawa, Y., and Kawagoe, R. (2000). Role of the basal ganglia in the control of purposive saccadic eye movements. Physiol. Rev. 80, 953-978.

Hutchison, W. D., Dostrovsky, J. O., Walters, J. R., Courtemanche, R., Boraud, T., Goldberg, J., and Brown, P. (2004). Neuronal oscillations in the basal ganglia and movement disorders: evidence from whole animal and human recordings. J. Neurosci. 24, 9240-9243.

Ibáñez-Sandoval, O., Carrillo-Reid, L., Galarraga, E., Tapia, D., Mendoza, E., Gomora, J. C., Aceves, J., and Bargas, J. (2007). Bursting in substantia nigra pars reticulata neurons in vitro: possible relevance for Parkinson disease. J. Neurophysiol. 98, 2311-2323.

Ibáñez-Sandoval, O., Hernandez, A., Floran, B., Galarraga, E., Tapia, D., Valdiosera, R., Erlij, D., Aceves, J., and Bargas, J. (2006). Control of the subthalamic innervation of substantia nigra pars reticulata by D1 and D2 dopamine receptors. J. Neurophysiol. 95, 1800-1811.

Kita, H. (2007). Globus pallidus external segment. Prog. Brain Res. 160, 111-133.

Kravitz, A. V., Freeze, B. S., Parker, P. R., Kay, K., Thwin, M. T., Deisseroth, K., and Kreitzer, A. C. (2010). Regulation of parkinsonian motor behaviours by optogenetic control of basal ganglia circuitry. Nature 466, 622-626.

Lee, C. R., and Tepper, J. M. (2007). A calcium-activated nonselective cation conductance underlies the plateau potential in rat substantia nigra GABAergic neurons. J. Neurosci. 27, 6531-6541.

Magill, P. J, Bolam, J. P., and Bevan, M. D. (2001). Dopamine regulates the impact of the cerebral cortex on the subthalamic nucleus-globus pallidus network. Neuroscience 106, 313-330.
Marshall, J. F., Henry, B. L., Billings, L. M., and Hoover, B. R. (2001). The role of the globus pallidus D2 subfamily of dopamine receptors in pallidal immediate early gene expression. Neuroscience 105, 365-378.

Matuszewich, L., and Yamamoto, B. K. (1999). Modulation of GABA release by dopamine in the substantia nigra. Synapse 32, 29-36.

Miyazaki, T., and Lacey, M. G. (1998) Presynaptic inhibition by dopamine of a discrete component of GABA release in rat substantia nigra pars reticulata. J. Physiol. 513, 805-817.

Morris, M. E., Iansek, R., Matyas, T.A., and Summers, J. J. (1994). The pathogenesis of gait hypokinesia in Parkinson's disease. Brain 117, 1169-1181.

Murray, A. M., Ryoo, H. L., Gurevich, E., and Joyce, J. N. (1994). Localization of dopamine D3 receptors to mesolimbic and D2 receptors to mesostriatal regions of human forebrain. Proc. Natl. Acad. Sci. U.S.A. 91, 11271-11275.

Nakanishi, H., Kita, H., and Kitai, S. T. (1987). Intracellular study of rat substantia nigra pars reticulata neurons in an in vitro slice preparation: electrical membrane properties and response characteristics to subthalamic stimulation. Brain Res. 437, 45-55.

Paxinos, G., and Watson, C. (1982). The Rat Brain in Stereotaxic Coordinates. New York: Academic Press.

Porceddu, M. L., Giorgi, O., Ongini, E., Mele, S., and Biggio, G. (1986). $3 \mathrm{H}-\mathrm{SCH} 23390$ binding sites in the rat substantia nigra: evidence for a presynaptic localization and innervation by dopamine. Life Sci. 39, 321-328.

Radnikow, G., and Misgeld, U. (1998). Dopamine D1 receptors facilitate GABAA synaptic currents in the rat substantia nigra pars reticulata. $J$. Neurosci. 18, 2009-2016.

Redgrave, P., Rodriguez, M., Smith, Y., Rodriguez-Oroz, M. C., Lehericy, S., Bergman, H., Agid, Y., DeLong, M. R., and Obeso, J.A. (2010). Goal-directed and habitual control in the basal ganglia: implications for Parkinson's disease. Nat. Rev. Neurosci. 11, 760-772.

Reiner, A., Medina, L., and Veenman, C. L. (1998). Structural and functional evolution of the basal ganglia in vertebrates. Brain Res. Rev. 28, 235-285.

Rivera, A., Trías, S., Peñafiel, A., Angel Narváez, J., Díaz-Cabiale, Z., Moratalla, R., and de la Calle, A. (2003). Expression of D4 dopamine receptors in striatonigral and striatopallidal neurons in the rat striatum. Brain Res. 989, 35-41.

Rivlin-Etzion, M., Marmor, O., Heimer, G., Raz, A., Nini, A., and Bergman, H. (2006). Basal ganglia oscillations and pathophysiology of movement disorders. Curr. Opin. Neurobiol. 16, 629-637.

Seeman, P., Wilson, A., Gmeiner, P., and Kapur, S. (2006). Dopamine D2 and D3 receptors in human putamen, caudate nucleus, and globus pallidus. Synapse 60, 205-211.

Smith, Y., Bevan, M. D., Shink, E., and Bolam, J. P. (1998). Microcircuitry of the direct and indirect pathways of the basal ganglia. Neuroscience 86, 353-387.

Smith, Y., and Bolam, J.P. (1989). Neurons of the substantia nigra reticulata receive a dense GABA-containing input from the globus pallidus in the rat. Brain Res. 493, 160-167.

Smith, Y., and Bolam, J. P. (1991). Convergence of synaptic inputs from the striatum and the globus pallidus onto identified nigrocollicular cells in the rat: a double anterograde labelling study. Neuroscience 144, 45-73.

Takakusaki, K., Habaguchi, T., OhtinataSugimoto, J., Saitoh, K., and Sakamoto, T. (2003). Basal ganglia efferents to the brainstem centers controlling postural muscle tone and locomotion: a new concept for understanding motor disorders in basal ganglia dysfunction. Neuroscience 119, 293-308.

Takakusaki, K., Saitoh, K., Harada, H., and Kashiwayanagi, M. (2004). Role of basal ganglia-brainstem pathways in the control of motor behaviors. Neurosci. Res. 50, 137-151.

Taverna, S., Canciani, B., and Pennartz, C.M.(2005). Dopamine D1-receptors modulate lateral inhibition between principal cells of the nucleus accumbens. J. Neurophysiol. 93, 1816-1819.

Tecuapetla, F., Carrillo-Reid, L., Guzmán, J. N., Galarraga, E., and Bargas, J. (2005). Different inhibitory inputs onto neostriatal projection neurons as revealed by field stimulation. $J$. Neurophysiol. 93, 1119-1126.

Wallmichrath, I., and Szabo, B. (2002). Cannabinoids inhibit striatonigral GABAergic neurotransmission in the mouse. Neuroscience 113, 671-682.

Walters, J. R., and Bergstrom, D. A. (2009). "Basal ganglia network synchronization in animal models of Parkinson's disease," in Cortico-Subcortical Dynamics in Parkinson's Disease Contemporary Neuroscience, ed. K.-Y. Tseng (New York: Humana Press), 117-142.

Walters, J. R., Hu, D., Itoga, C. A., ParrBrownlie, L. C., and Bergstrom, D. A. (2007). Phase relationships support a role for coordinated activity in the indirect pathway in organizing slow oscillations in basal ganglia output after loss of dopamine. Neuroscience 19, 762-776. 
Yanovsky, Y., Mades, S., and Misgeld, U. (2003). Retrograde signaling changes the venue of postsynaptic inhibition in rat substantia nigra. Neuroscience 122, 317-328.

Zhou, F. W., Jin, Y., Matta, S. G., Xu, M., and Zhou, F. M. (2009). An ultrashort dopamine pathway regulates basal ganglia output. J. Neurosci. 29, 10424-10435.

Zold, C. L., Belluscio, M., Kasanetz, F., Pomata,P.E., Riquelme, L.A., Gonon, F., and Murer, M. G. (2009). "Converging into a unified model of Parkinson's disease pathophysiology," in CorticoSubcortical Dynamics in Parkinson's Disease Contemporary Neuroscience, ed. K.-Y. Tseng (New York: Humana Press), 252-259.

Conflict of Interest Statement: The authors declare that the research was conducted in the absence of any commercial or financial relationships that could be construed as a potential conflict of interest.

Received: 15 December 2010; paper pending published: 04 January 2011; accepted: 23 January 2011; published online: 10 February 2011.

Citation: Aceves JJ, Rueda-Orozco PE, Hernández R, Plata V, Ibañez-Sandoval O, Galarraga E and Bargas J (2011) Dopaminergic presynaptic modulation of nigral afferents: its role in the generation of recurrent bursting in substantia nigra pars reticulata neurons. Front. Syst. Neurosci. 5:6. doi: 10.3389/fnsys.2011.00006

Copyright (c) 2011 Aceves, Rueda-Orozco, Hernández, Plata, Ibañez-Sandoval, Galarraga and Bargas. This is an openaccess article subject to an exclusive license agreement between the authors and Frontiers Media SA, which permits unrestricted use, distribution, and reproduction in any medium, provided the original authors and source are credited. 